J. Phys. IV France 133 (2006) 339-342

(C) EDP Sciences, Les Ulis

DOI: 10.1051/jp4:2006133068

\title{
Kinetic effects in stimulated Brillouin scattering
}

\author{
F. Detering ${ }^{1}$, J.-C. Adam ${ }^{1}$, A. Heron ${ }^{1}$, S. Hüller ${ }^{1}$, P.-E. Masson-Laborde ${ }^{1}$ \\ and D. Pesme ${ }^{1}$
}

${ }^{1}$ CPHT, École Polytechnique, CNRS UMR 7644, 91128 Palaiseau Cedex, France

\begin{abstract}
The role of ion and electron kinetic effects in the nonlinear evolution of stimulated Brillouin scattering (SBS) is investigated by means of particle-in-cell numerical simulations. The simulations were carried out in one and two spatial dimensions (1D and 2D), with a full PIC code, in which both ions and electrons are kinetic. The full PIC simulations are compared with those obtained from a hybrid PIC code (kinetic ions and Boltzmann electrons), making it possible to determine in which limit the electron kinetic effects are important. The simulation geometry corresponds to a coherent laser beam interacting with an expanding plasma slab. In the 1D simulations, the interaction becomes incoherent, as time goes on, in a domain that spatially begins in the plasma region close to the laser light entrance, and that ends within the plasma at a frontier which moves faster than the ion acoustic wave (IAW) velocity. The higher the laser intensity, the faster moves the frontier of this spatial domain. The SBS reflectivity drops at the very moment when this domain fills entirely the plasma. Two regimes have to be distinguished. In the regimes of low laser intensity, strong sub-harmonic generation of the excited IAW is observed to take place in this moving spatial domain, so that the SBS reflectivity drop is interpreted as being due to sub-harmonic generation. In the opposite regime of high laser intensity, there is no evidence of strong sub-harmonic generation, whereas a strong ion heating is observed, so that the reflectivity drop is interpreted as being due to enhanced ion damping. In the 1D simulations the electron kinetic effects are found to be able to smooth temporally the SBS reflectivity, although the overall picture remains the same when the electrons are taken as a Boltzmann fluid. In the 2D simulations, the SBS reflectivity is observed to drop rapidly in time because of the efficient nonlinear Landau damping on the ions, as previously reported by Cohen et al. [1]. In these 2D simulations, the electron kinetic effects are found to play a negligible role as compared with the ion kinetic effects.
\end{abstract}

\section{INTRODUCTION}

This paper is concerned with the stimulated Brillouin scattering (SBS) instability, that is the process by which the incident laser wave couples with an ion acoustic wave (IAW) to give rise to a scattered transverse wave. It is now widely admitted that the low reflectivity observed in many present day experiments SBS could be explained only if the kinetic effects are properly taken into account in the SBS modeling. In the regime of partial particle trapping, the kinetic effects give rise to a nonlinear frequency shift (NLFS) to the IAW [2]. In the regimes of moderate linear SBS gain factors, the spatial inhomogeneity of this NLFS plays the same role as a flow inhomogeneity, and consequently reduces non-linearly the SBS gain factor [3]. On the other hand, the fundamental IAW component can be unstable with regards to the so called two-ion decay, which is a parametric instability triggered by the IAW nonlinearity. Depending on the sign and size of the NLFS, this parametric instability can be enhanced or quenched [4]. It is therefore of fundamental importance to investigate the role of kinetic effects on the SBS behaviour. In the present contribution we report on results of extensive numerical simulations carried out with our code Kolipic [5], which is a hybrid PIC code similar to BZOHAR [6, 7], in which the ions are kinetic, the electrons form a Boltzmann fluid, the longitudinal electric field is obtained by solving the Poisson equation, the transverse EM fields are described by a time enveloped wave equation without the paraxial approximation. In order to investigate the role of the electron kinetic effects, we compare the hybrid PIC results with those obtained from full PIC code, in which both ions and electrons are kinetic and the Maxwell equations are solved. 


\section{NUMERICAL SIMULATIONS OF SBS IN AN EXPANDING PLASMA}

\subsection{System parameters}

All the simulations were carried out in the geometry of an expanding plasma foil. The initial profile was a homogeneous plasma sheath of thickness $L_{p}=728 k_{0}^{-1}$, bordered on each side by a vacuum zone in which the plasma expands. Here $k_{0}$ denotes the laser light wave vector in vacuum. The plasma parameters were the following: the ions were hydrogen with $Z=1$, the electron temperature was $T_{e}=1 \mathrm{keV}$, the electron density was varied in the range $0.03<n / n_{c}<0.7$ in order to investigate the $k_{s} \lambda_{D e}$ dependence, $k_{s}$ denoting the wave-number of the fundamental IAW component. The electron-ion temperature ratio $T_{e} / T_{i}$ was varied in the range $12<T_{e} / T_{i}<18$ in order to consider differently damped regimes; this range corresponds to a tenfold increase in the linear ion Landau damping. In the one-dimensional simulations, the laser intensity was varied in the interval $[1-10] 10^{14} \mathrm{~W} / \mathrm{cm}^{2}$.

\subsection{Low and high intensity regimes in 1D hybrid simulations}

The intensity scan in the 1D simulations presented here, $T_{e} / T_{i}=18$ and $k_{s} \lambda_{D}=0.25$, reveals significantly different SBS behaviours between those carried out with the lowest intensities $\left(1-2.510^{14} \mathrm{~W} / \mathrm{cm}^{2}\right)$ and those carried out with the highest ones $\left(5-1010^{14} \mathrm{~W} / \mathrm{cm}^{2}\right)$.

The low intensity regime is characterized by a strong subharmonic generation. Subharmonic generation coincides with the first decline of the instantaneous reflectivity, as can be clearly seen in the left of Figure 1. By Fourier analyzing the IAW in subdomains corresponding to moving spatial windows, we found out that, initially, subharmonic generation takes place in a small region close to the laser light entrance. The generation gives rise to a decay of the excited IAW. In the resulting zone of incoherence, which is defined by a broad spectrum as opposed to only harmonics, SBS does no longer grow. Therefore, SBS can develop only in a zone deeper into the plasma. This process keeps on repeating, so that the zone of incoherence grows with time, its internal frontier traveling much faster than the ion sound, until it reaches the plasma boundary where the laser light exits. At this time, the reflectivity drops to the percent level. This reflectivity drop occurs well before the hydrodynamic background evolution is able to reduce the SBS growth, as observed previously for a different set of parameters and attributed to the frequency shift inhomogeneity [3].

In the high intensity regime, the final reflectivity drop also occurs at the very moment when the region of IAW incoherence reaches the exit plasma boundary. The main differences as compared with the low
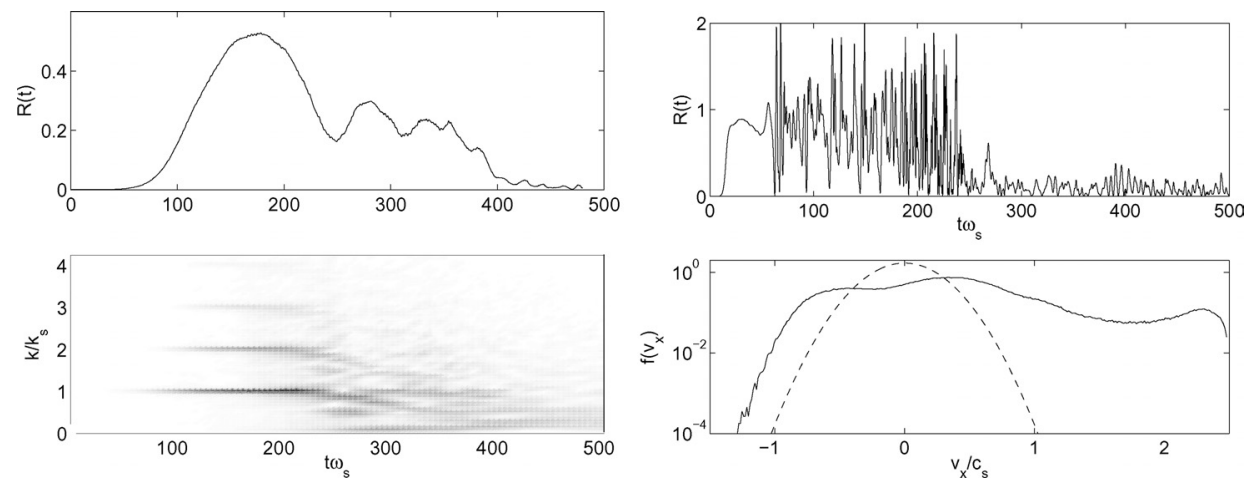

Figure 1. Left : Reflectivity for $I_{14} \lambda^{2}=1$ (top panel), IAW spectrum $\Phi_{k}$ as a function of time in a window moving with $c_{s}$ of width $24 \lambda_{s}$ near the plasma entrance (bottom panel). Right : Reflectivity at $I_{14} \lambda^{2}=10$ (top panel), ion velocity distribution at $t \omega_{s}=25$ (initial distribution in dashed line) near the entrance averaged over a zone of $29 \lambda_{s}$ (bottom panel). 
intensity case are: (i) this process develops much faster, (ii) it is accompanied by very large bursts in the reflected light, as can be seen on the right, top panel of Figure 1, (iii) the fundamental IAW component does not decay with time to vanishing levels, its energy remaining of same order of magnitude as the one contained in all the subharmonics, (iv) there is a strong ion heating and tail formation in the ion distribution function, as can be seen in the right, bottom panel of Figure 1.

\subsection{Electron kinetic effects in 1D: full PIC simulations}

We carried out simulations with the full PIC code and for the same geometry as for the hybrid ones in order to investigate the relevance of the electron kinetic effects [4]. They were carried out with $n / n_{c}=0.4$ and $T_{e}=8 \mathrm{keV}$ in order to suppress Raman scattering while maintaining the same high value for the parameter $k_{s} \lambda_{D} \approx 0.25$.

The full PIC simulations results show that the main effect of the electron kinetic effects in 1D is a temporal smoothing of the instantaneous reflectivity, that manifests by the disappearance of the high reflectivity bursts at high intensity. On the other hand, the average evolutions of the reflectivity are very similar in both types of simulations, in the range of laser intensities considered here. By contrast, in the regime of very high intensities, the behaviour is very different between the two kinds of simulations [8].

\subsection{Full PIC simulations in 2D}

A two-dimensional plane wave simulation carried out with the laser intensity $I=2.510^{14} \mathrm{~W} / \mathrm{cm}^{2}$ demonstrates the importance of the transverse effects, by comparison with the 1D results, as shown in Figure 2. A significant amount of energy is transferred in the transverse motion and results in an effective heating of the velocity distribution. This energy transfer limits the reflectivity at a lower level and causes it to descend faster than in the 1D simulations, as already pointed out in Ref. 1.

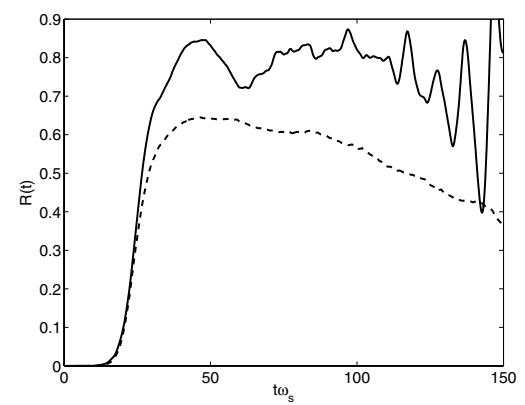

Figure 2. Reflectivities at $I_{14} \lambda^{2}=2.5$ of $1 \mathrm{D}$ (solid) line and 2D (dashed line) plane wave hybrid PIC.

The comparison of results of the 2D simulations carried out with the hybrid PIC code and those with the full PIC code for $n / n_{c}=0.3, T_{e}=2 \mathrm{keV}$ and $I=10^{15} \mathrm{~W} / \mathrm{cm}^{2}$ shows a very close agreement, indicating that in this regime the transverse effects associated with ion kinetic effects dominate over the electron kinetic effects, so that the latter can be ignored in $2 \mathrm{D}$.

\section{References}

[1] B. Cohen, L. Divol, A. B. Langdon, and E. A. Williams, Physics of Plasmas 12, 052703 (2005).

[2] G. J. Morales and T. M. O’Neil, Phys. Rev. Lett. 28, 417 (1972).

[3] L. Divol, B. Cohen, A. B. Langdon, and B. F. Lasinski Physics of Plasmas 10, 3728 (2003). 
[4] C. Riconda, A. Heron, D. Pesme, S. Hüller, V. T. Tikhonchuk, and F. Detering, Phys. Rev. Lett. 94, 055003 (2005); D. Pesme, C. Riconda, and V. T. Tikhonchuk, Physics of Plasmas 12, 092101 (2005).

[5] C. Riconda, S. Hüller, J. Myatt, and D. Pesme, Phys. Scr. 84, 217 (2000).

[6] B. Cohen, B. F. Lasinski, A. B. Langdon, and E. A. Williams, Physics of Plasmas 4, 956 (1997).

[7] B. Cohen, B. F. Lasinski, A. B. Langdon, E. A. Williams, and H. A. Baldis, Physics of Plasmas 5, 3402 (1998).

[8] S. Weber, C. Riconda, and V. T. Tikhonchuk, Phys. Rev. Lett. 94, 055005 (2005). 